\title{
Big Data Analytics in Healthcare and Delve Bioinformatics Data Space for Health Amelioration
}

\author{
Gakwaya Nkundimana Joel \\ PhD Scholar, CS Dept. \\ Karpagam Academy of Higher Education \\ Coimbatore-21 \\ India
}

\author{
S. Manju Priya, $\mathrm{PhD}$ \\ Associate Professor, CS, CA \&IT \\ Karpagam Academy of Higher Education \\ Coimbatore-21 \\ India
}

\begin{abstract}
The healthcare informatics focuses on health data, information and knowledge, including their collection, processing, analysis and use bioinformatics employs computational tools and techniques to study and analyze large biological databases and to understand disease and study of inherent genetic information molecular structure by relating them with healthcare data. This amassing of healthcare information will enable the biologist and scientist to improve health as drug discovery. This paper touches on big data in healthcare and analyzes of those big data in healthcare for the better improvement of healthcare system, bioinformatics data stored in secured manner. Finally, the paper looks on the helpful result, the beneficence of each of them in amelioration of healthcare system. To achieve this health amelioration in bioinformatics, we use Hadoop as tools which collect and analyze the huge amount of data in healthcare system.
\end{abstract}

\section{Keywords}

Big data, Data Analytics, healthcare, bioinformatics, Hadoop.

\section{INTRODUCTION}

We as Data mining is a study of delving the concealed data in a database and is also known as analysis steps of knowledge discovery in database [1]. Techniques of data mining help to process the data and turn them into useful information. Prediction results from data mining are useful invarious fields like Business Intelligence, Bioinformatics, Healthcare Management, Finance etc. Medical field has wide amount as well as variety of data for processing and there exist many challenging tasks [13].

Data mining aspires to draw forth information from dataset and remodel it so that the meaning of those data will be ease understandable. The huge amount of data collected in data mining is termed as big data. Big data is a term that can be described at least three separate, but interrelated trends: capturing and managing lots of information, working with many new types of data and exploiting these masses of information and new data types.

This data mining covers the whole part of the education disciplines such as biological data, mathematical, physical, medicinal etc. Study that marvelous organism, the human system, and the laws by which it is governed is one of the earthshaking area of economy; as such for a society to realize its dream and vision of sustainable growth, it is very important to know that every careless action, any abuse put upon the wonderful mechanism, by disregarding his specified condition the future of nation will suffer. For this precious life there is a saying among society which says that "health is wealth" [2].
The analytics in healthcare come as a result of large healthcare data that are being gathered electronically. Therefore, any technology or system is acclimated to improve the healthcare system. The recent human Genome Project has found that the opportunities come to be able to develop such data, analyze, and present the genomic data in a quicker, cheaper and reliable way. This grand technological advance has affected healthcare in condition of new prevention, development, diagnosis, and ameliorated treatment for regular healthcare. These huge amount of data collected from bioinformatics and healthcare informatics fields paired with analytics are appraised to save in near future preventive, predictive and personalized healthcare aids. For this high appraisal of analytics of big data in healthcare give tremendous aid to national prosperity and development.

\subsection{Big Data}

Big data is a current voluminous technology which is convoluted to deal with the existing databases due to contain and collect dissimilar data from various sources. Such huge amount of data become very difficult to process them [3]. Different approaches have been introduced to handle the stored data in healthcare system. The incessant augmenting of number of people, technology, foods, air pollution, etc. the huge issue of treating and preventing disease among nation requires the studied and careful strategic manner. This is really essential to keep the nation of worldwide safe from maladies without cure. Healthcare systems receive and store the huge amount of data where the interpretation and the result of tests require new tools which can process and interpret data in short time and efficient manner. By providing the exact result in healthcare system drug discovery will be opened the door.

\section{BIG DATA CHALLENGE IN HEALTH}

In healthcare the difficulties are encountered in interpreting heavy data. The bigness of data has serious meaning in processing and providing the accurate result. This trends are caused by increase of different devices which are developed day to day in healthcare to ameliorate the health, for this cause the data traffic in healthcare system has become challenge. Diversity data in health, assortment of data begin to occur as a fact to join the different assort of biomedical data sources available. The swiftness of data processing is considered as one of the challenge, manifoldness of the data captured in different devices is also one the fact the call in healthcare, furthermore data are produced tremendously.

\subsection{Management issue}

Most preeminent thing to deal in technology industries is known as big data and its management. The way data are collected in large number from different fountainhead of 
technologies cause some ordinary tools to fail their intended purpose of creation. The action of controlling and way of deciding what to do is what all companies and industries need in order to advance their work and better service to their clients.

\subsection{Storage issue}

In order to provide the best captured images or video information we need the high definition tools, this tools capture the data with big size, the storage of this data will be an issue to tackle. There are many company which store the big data in healthcare system this storage will need another tools which can reduce the data without losing its quality. The traditional storage in health lack the capacity of storing the variety of data and a tool which can analyze those data for the medication discovery and disease prevention.

\section{BIOINFORMATICS WITH ITS BENEFACTION}

The lore of studying how intricate biological data are analyzed and collected is known as bioinformatics. Some specialized computer software is used in bioinformatics in order to apprehend the biological data. In this study, an inborn genetic disease computer is developed with the desire of meeting the collection and anatomizing of biological data. The first tool was BLAST (Basic Alignment Search Tools) [5] it is used to examine new DNA or Protein sequence. BLAST compare new sequence to all sequence in database to find the most similar known sequence.

\subsection{Delve bioinformatics data space}

To delve in bioinformatics is in another word to search hidden information from the stored data. The healthcare information deals with the best use of information, through the help of technology, in ameliorating healthcare, public health. When the data are delved correctly it gives the information about a patient's state of health to right person, at the right time it is required, thereby guiding them in making and taking informed decisions about patient's treatment. Medical informatics makes available the essential tools to relate data and knowledge in the process of decision making. Information management and technology ensures collection from various sources, as well as processing and delivery of the same. The main aim of this delve data space in bioinformatics is to increase the performance of treatment because the information has interpreted correctly.

\subsection{Healthcare analytics}

Analytics serve as one of the key central of all decision making. From a careful analysis in healthcare a decision concerning particular disease medication will be made and help the patients to improve their health. The big data guarantees the support of a large number of medical and healthcare which needs to be analyzed so that they can support disease surveillance and population health management. It is also used to obtain various data from patient, patient being able to access their own data and personal health records and finally being able to work across these multiple data to identify patient and population health needs. The best use of healthcare analytics will provide the achievement of the best output result. Analytics will provide therefore ameliorate efficiency, aid discovery and exploration of new drugs and advanced treatment.

\section{HADOOP}

Hadoop is a java Framework which has developed to handle the large and complicated data from different distributed sources at minimum cost [6]. Stored data in Hadoop is done by the help of things called Master gobbet and Slave gobbet. The master gobbet acts as Chief of the Hadoop where on the other hand the slave gobbet serve as Servant. The Chief gobbet known as master contains the information related to all servant gobbet(slave). The servant gobbets are intended to executed the given task from the chief gobbet.

\subsection{Hadoop distributed file and Map- Reduce}

The title (Helvetica 18-point bold), authors' names (Helvetica Hadoop Distributed File System came as solution to the network distributors. The files were distributed but their storage was an issue. In Hadoop ecosystem it provides the operation of reading many times as long as you wrote it once [7]. Accumulation of files are done in form of block with a nonfeasance of 64MB. Map Reduces one of the core component of Hadoop which is made for processing of large volume of data. In order to drive huge amount of data we need Map Reduce. This Map Reduce is divided into three:

$$
\begin{array}{ll}
\text { 1. } & \text { Mapper } \\
\text { 2. } & \text { Sort and Shuffle } \\
\text { 3. } & \text { Reduce }
\end{array}
$$

12-point) and affiliations (Helvetica 10-point) run across the full width of the page - one column wide. We also recommend e-mail address (Helvetica 12-point). See the top of this page for three addresses. If only one address is needed, center all address text. For two addresses, use two centered tabs, and so on. For three authors, you may have to improvise.

\section{RELATED WORK}

Place Tables/Figures/Images in text as close to the reference

Here we are going to see the reviewed current research and the approach used to improve health in healthcare system

In [8], the author introduced tools of handling big data in healthcare where we have different sources of information this tool helps the advancement of big data in providing the more awareness of diseases and way of preventing them. In his research he has proposed different tools such as EHRs (Electronics Health Records) to gather and store the patient data. Social health is also seen as one of the big data holders where a patient is connected to the doctor on the far side of clinic with involvement of social networks. Here patients can communicate on their health. These social health supply big data.

In processing those big data, Hadoop is needed to process and store large volume of data. The security of data transmission and storage is the most downside.

In [9], the author has introduced a way of handling big data in a secured manner from unexpected leakage of data and from the data hackers to collect public and private policy resources whenever they want. Three methods of securing big data have been introduced:

\section{Kerbros, 2. Algorithm, 3. NameNode}

Kerbros is one of the method suggested to protect the sent data across a network especially in providing firm genuine secret-codes.The connection in Hadoop storage between its master node and slave node is established with the help of Remote Procedure Call(RPC). The Kerbros is used to protect a RPC. If a client wants to access the data from slave the permission is granted by master to ensure which slave is requesting the wanted file. The advantage of this paper is to 
be implemented in healthcare system, to authenticate the Social health system.

In [10], the author has used machine learning to handle big data in a way of analysis the huge data from bioinformatics by using the interaction components computing technology. Most of the tools which are used to handle big data use processing without manual interaction. The current tools use in bioinformatics such as Co-Expression and regulatory needs to test DNA and RNA and to process Protein episodically and swiftly states the disease in heterogeneous network. The downside of these above tools, they have lack of standard big data architecture.

In [11], the author introduced healthcare informatics on health data, the contribution of bioinformatics is to find and gather the huge amount of data, the healthcare informatics to provide the right and useful information about patient's state of health and the management of healthcare facilities. This healthcare helps in improvement that facilitate the quality of healthcare. The analytics process was made to ensure the healthcare management which compare health and fitness. The decision about treatment and support is also improved

In [12], author has introduced the increment of population which is proportional to the crime, lawbreaking standard concerning facts that has be acted is an enormous outcome that the states and administrations are focusing on in order to affirm that the committed acts are adjudicated justly. This analysis leads to Big Data Analytics. The method which has been used in crime data analysis is PIG. In the sector of medical field, it can also be applied to analyze the huge amount data.as possible (see Figure 1). It may extend across both columns to a maximum width of $17.78 \mathrm{~cm}(7$ ').

Captions should be Times New Roman 9-point bold. They should be numbered (e.g., "Table 1" or "Figure 2"), please note that the word for Table and Figure are spelled out. Figure's captions should be centered beneath the image or picture, and Table captions should be centered above the table body.

\section{CONCLUSION}

It has been shown that biology and technology in the term of bioinformatics is to provide the opportunity for back and forth in technology, data, and knowledge. This will help in determining the database of diseases, their causes, the opened way of treating those disease and cure which will prevent the spread of those maladies. By using Hadoop framework, the analytical point of view in bioinformatics will be improved and the life of nation will be ameliorated through the reduction of disease, identify future diagnostics. The future work will be of securing Hadoop in bioinformatics and its improvement.

\section{REFERENCES}

[1] Kmiecik, Sebastian; Gront, Dominik; Kolinski, Michal; Wieteska, Lukasz; Dawid, Aleksandra Elzbieta; Kolinski, Andrzej (2016-06-22). "Coarse-Grained Protein Models and Their Applications". Chemical Reviews. doi:10.1021/acs.chemrev.6b00163. ISSN 0009. 2665.

[3] Reichman, O.J.; Jones, M.B.; Schildhauer, M.P. (2011). "Challenges and Opportunities of Open Data in Ecology". Science. 331 (6018): 703-5. doi:10.1126/science.1197962. PMID 21311007.

[4] www. Edureka.co/

[5] Christopher Matthews (26 April 2013). "Trouble With Your Investment Portfolio? Google It!". TIME Magazine. Retrieved 9 August 2013.

[6] Philip Ball (26 April 2013). "Counting Google searches predicts market movements". Nature. Retrieved 9 August 2013.

[7] Bernhard Warner (25 April 2013). "'Big Data' Researchers Turn to Google to Beat the Markets". Bloomberg Businessweek.

[8] "'Big Data' Researchers Turn to Google to Beat the Markets". Bloomberg Businessweek. Retrieved 9 August 2013.

[9] [8] Javier Andreu-Perez, Carmen C.Y. Poon, "Big data for Health",IEEE journal of biomedical and health Informatics, Vol. 19, No. 4, July 2015

[10] B. Saraladevi, N. Pazhaniraja, P. Victer Paul, "Big Data and Hadoop-A Study in Security Perspective", 2nd International Symposium on Big Data and Cloud computing(ISBCC'15)

[11] Hirak Kashyap, Hasin Afzal Ahmed "Big Data Analytics in Bioinformatics: A Machine Learning Prespective", Journal of latex Class Files, Vol. 13, No. 9, September 2014

[12] Jelili Oyelade, Jumoke Soyeni "Bioinformatics, Healthcare Informatics and Analytics: An imperative for Improvement Healthcare System", International Journal of Applied Information System( IJAIS) - ISSN: 2249-0868 Foundation of Computer Science FCS, New York, USA Vol. 8, No. 5, February 2015

[13] Arushi Jain, Vishal Bhatnagar "Crime Data Analysis Using Pig with Hadoop", International Conference on information Security \& Privacy(ICISP2015), 11-12 December 2015, Nagpur, India

[14] V.Kirubha, S.Manju Priya, "Survey on Data Mining Algorithms in Disease Prediction", International Journal of Computer Trends and Technology (IJCTT) - Volume 38 Number 3 - August 2016 